\title{
Mode of frequency distribution of external work efficiency of arrhythmic beats during atrial fibrillation remains normal in canine heart
}

\author{
Juichiro Shimizu • Satoshi Mohri · Haruo Ito • \\ Miyako Takaki · Hiroyuki Suga
}

Received: 10 September 2008/ Accepted: 5 November 2008/Published online: 12 December 2008

(C) The Physiological Society of Japan and Springer 2008

\begin{abstract}
The external work (EW) efficiency of individual arrhythmic beats of the left ventricle (LV) cannot directly be obtained since $\mathrm{LV} \mathrm{O}_{2}$ consumption $\left(\mathrm{VO}_{2}\right)$ of each beat cannot directly be measured under beat-to-beat varying contractile and loading conditions. We, however, have recently reported that $\mathrm{VO}_{2}$ of each arrhythmic beat can reasonably be estimated by $V \mathrm{O}_{2}=a \mathrm{PVA}+b \mathrm{E}_{\max }+c$ even under varying PVA and $\mathrm{E}_{\max }$. Here, PVA is the LV pressure-volume $(P-V)$ area as a measure of the LV total mechanical energy, $E_{\max }$ is the $\mathrm{LV}$ end-systolic elastance as an index of the LV contractility, $a$ is a constant $\mathrm{O}_{2}$ cost of PVA, $b$ is a constant $\mathrm{O}_{2}$ cost of $\mathrm{E}_{\max }$, and $c$ is the basal metabolic $V_{2}$ of the beat, all on a per-beat basis. Using the above formula in this study, we calculated $V_{2}$ of the individual arrhythmic beats from their measured PVA and $\mathrm{E}_{\max }$ during electrically induced atrial fibrillation (AF) in normal canine hearts. We then calculated their LV EW efficiency by dividing their measured EW with the estimated $V \mathrm{O}_{2}$. We found that the thus calculated EW efficiency of the arrhythmic beats had a rightward skewed distribution with a mode of $15 \%$ and a maximum of $18 \%$ around a mean of $13 \%$ on average in six hearts. This mode remained comparable to the efficiency $(15 \%)$ at regular
\end{abstract}

J. Shimizu $(\bowtie) \cdot$ H. Ito $\cdot$ M. Takaki

Department of Physiology II, Nara Medical University,

Shijocho, Kashihara, Nara 634-8521, Japan

e-mail: juichiro@naramed-u.ac.jp

J. Shimizu $\cdot$ S. Mohri $\cdot$ H. Suga

Department of Systems Physiology, Okayama University

Graduate School of Medicine, Dentistry, and Pharmaceutical

Sciences, Shikatacho, Okayama 700-8558, Japan

H. Suga

National Cardiovascular Center Research Institute, Fujishirodai,

Suita, Osaka 565-8565, Japan tachycardia though $22 \%$ lower than mean arrhythmic tachycardia.

Keywords Arrhythmia - Cardiac efficiency $\cdot \mathrm{E}_{\max }$. Oxygen consumption $\cdot$ PVA

\section{Introduction}

We have already established that left ventricular (LV) $\mathrm{O}_{2}$ consumption $\left(V_{2}\right)$ is reasonably predictable by $V \mathrm{O}_{2}=$ $a \mathrm{PVA}+b \mathrm{E}_{\max }+c$ on a per-beat basis in regular beats $[1,2]$. Here, PVA is the LV pressure-volume $(P-V)$ area as a measure of the LV total mechanical energy [1], $E_{\max }$ is the LV end-systolic elastance or $P-V$ ratio as an index of contractility [3], $a$ is a constant $\mathrm{O}_{2}$ cost of PVA, $b$ is a constant $\mathrm{O}_{2}$ cost of $\mathrm{E}_{\max }$, and $c$ is the basal metabolic $V \mathrm{O}_{2}$, all per beat [1]. The above formula proved to hold in steady-state beats under stable LV contractile and loading conditions where $V \mathrm{O}_{2}$ per beat is simply equal to $V \mathrm{O}_{2}$ per min divided by the regular heart rate [1]. We previously extrapolated the above formula to individual arrhythmic beats and reported its feasibility to predict the respective $V \mathrm{O}_{2}$ on a per-beat basis even under atrial fibrillation (AF) [4]. The purpose of the present study is to determine whether the EW efficiency of individual beat under AF is reasonably predictable from the measured EW and the predicted $V \mathrm{O}_{2}$ /beat.

In the present study, we applied the above-established formula for the first time to estimate the external work (EW) efficiency of individual arrhythmic beats in six normal canine in situ LVs under electrically induced AF. We measured LV $\mathrm{E}_{\max }$ and PVA as well as EW of individual arrhythmic beats under AF. We then substituted these $\mathrm{E}_{\max }$ and PVA data into our $V \mathrm{O}_{2}$ formula shown above with the 
physiologically normal representative $a$ and $b$ values and the $c$ value as the basal metabolic $V \mathrm{O}_{2}$. We then calculated the EW efficiency of each individual arrhythmic beat by dividing the measured $\mathrm{EW}$ with the estimated $\mathrm{VO}_{2}$ of the beat.

As the result, we found that the EW efficiency under AF varied around a mean of $13 \%$, ranging between 5 and $18 \%$, on average. The distribution of the EW efficiency tended to skew to the higher side with a mode of $15 \%$ on average. Although the mean efficiency under AF was significantly smaller than the efficiency under regular tachycardiac pacing, the mode efficiency was comparable to the efficiency of the regular beat and the maximum efficiency exceeded it in the respective hearts. We reasonably accounted for these statistical results by the ratio of the normally distributing EW of each beat under AF to the respective $V \mathrm{O}_{2}$ linearly correlating with $\mathrm{EW}$, but always containing a considerable PVA-independent $V \mathrm{O}_{2}$ consisting of both basal metabolic $\mathrm{VO}_{2}$ and excitation-contraction coupling $\mathrm{VO}_{2}$.

\section{Methods}

\section{Surgical preparation}

We performed the canine experiments in conformity with the guiding principles for the care and use of animals in the field of physiological sciences of the Physiological Society of Japan and the American Physiological Society. We anesthetized six adult mongrel dogs (mean \pm SD: $8.6 \pm 1.0 \mathrm{~kg})$ with pentobarbital sodium $(25 \mathrm{mg} / \mathrm{kg}$, i.v. $)$ after premedication with ketamine hydrochloride $(50 \mathrm{mg} /$ $\mathrm{kg}$, i.m.) and intubated for artificial respiration in each experiment. We maintained the anesthesia by fentanyl $(100 \mu \mathrm{g} / \mathrm{h}$ per dog, i.v.) as usual in our laboratory [4].

After midsternal thoracotomy, we inserted a 3F cathetertip micromanometer into the LV from the apex to measure the LV pressure $(\mathrm{P})$. We introduced a 7F eight-electrode conductance catheter (Webster Laboratories, Baldwin Park, CA, USA) into the LV through an apical stab and placed it along the ventricular long axis to measure instantaneous LV volume (V) continuously. We described the method for measuring LVV with this catheter elsewhere [4].

Briefly, the catheter measured continuously the timevarying electrical conductance $G(t)$ of the blood within the LV cavity of the beating heart $[5,6]$. Our custom-made signal conditioner-processor (SI Medicotech Co., Ltd., Osaka, Japan) converted the G(t) to LV conductance volume after calibrating blood conductivity in the sampling cuvette. We obtained the parallel conductance Gp attributable to the conductance of the LV wall and the surrounding tissues and fluid by the standard hypertonic saline dilution method. From this Gp, we calculated a constant offset volume $(\mathrm{Vc})$ and obtained the absolute LVV by subtracting $\mathrm{Vc}$ from the LV conductance volume $[5,6]$.

We attached a pair of stimulation electrodes to the left atrial appendage. Suprathreshold electrical stimulation at $20 \mathrm{~Hz}$ via these electrodes induced and maintained AF. AF started and stopped the stimulation [5]. We maintained $\mathrm{AF}$ for $2 \mathrm{~min}$ and recorded LVP and LVV during the latter $1 \mathrm{~min}$ at sampling intervals of $3 \mathrm{~ms}$ in a computer (Fig. 1a). We also recorded LVP and LVV while pacing the left atrium at a constant rate for 2 min after we raised the regular pacing rate to approach the mean arrhythmic heart rate during AF until pulsus alternans barely started. As the result, the regularly paced tachycardiac heart rate (mean \pm SD: $170 \pm 17$ beats $/ \mathrm{min}$ ) reached $78 \%$ on average of the mean arrhythmic heart rate $(219 \pm 51$ beats/ $\min$ ) in the six hearts.

The LV, including the septum, weighed $46.5 \pm 15.1 \mathrm{~g}$ after each experiment. Using these LV weights, we normalized LV mechanoenergetic variables relative to $100 \mathrm{~g}$ in each $\mathrm{LV}$.

\section{Mechanoenergetic variables}

We obtained such key mechanoenergetic variables as EW, $\mathrm{E}_{\max }$, PVA, dead or unstressed volume $\left(V_{0}\right)$, mechanical potential energy (PE), stroke volume (SV), end-diastolic volume (EDV), and end-systolic pressure (ESP) of individual arrhythmic beats under electrically induced $\mathrm{AF}$ as well as regular stable tachycardiac beats in each LV. Figure 1 illustrates these variables under $\mathrm{AF}$ in the $P-V$ diagram. We have described in detail the methods to calculate EW, $\mathrm{E}_{\max }$, and PVA elsewhere [7, 8]. Briefly, we first obtained $V_{0}$ (Fig. 1) as the $\mathrm{V}$-axis intercept of the straight line drawn through the end-systolic $P-V$ points of the shrinking $P-V$ loops while clamping the inferior vena cava during 10-20 regular beats, as described elsewhere [1, 3]. We then obtained $L V E_{\max }$ as the slope of the line connecting $V_{0}$ and the left-upper, end-systolic corner of each $P-V$ loop (Fig. 1).

We also calculated PVA as usual on a computer as the $P-V$ area scanned by the instantaneous $P-V$ line connecting the instantaneous $P-V$ point to $V_{0}$ in each arrhythmic beat [7, 8]. This calculation also yielded EW and PE (shaded rectangular and triangular areas, respectively, in Fig. 1) as the two components of PVA. We normalized $\mathrm{E}_{\max }$, PVA, $\mathrm{EW}$, and PE relative to $100 \mathrm{~g} \mathrm{LV}$.

$V \mathrm{O}_{2}$ estimation

We estimated $V \mathrm{O}_{2}$ of each individual arrhythmic beat from the normalized $\mathrm{E}_{\max }$ and PVA by calculating it with the following equation: 

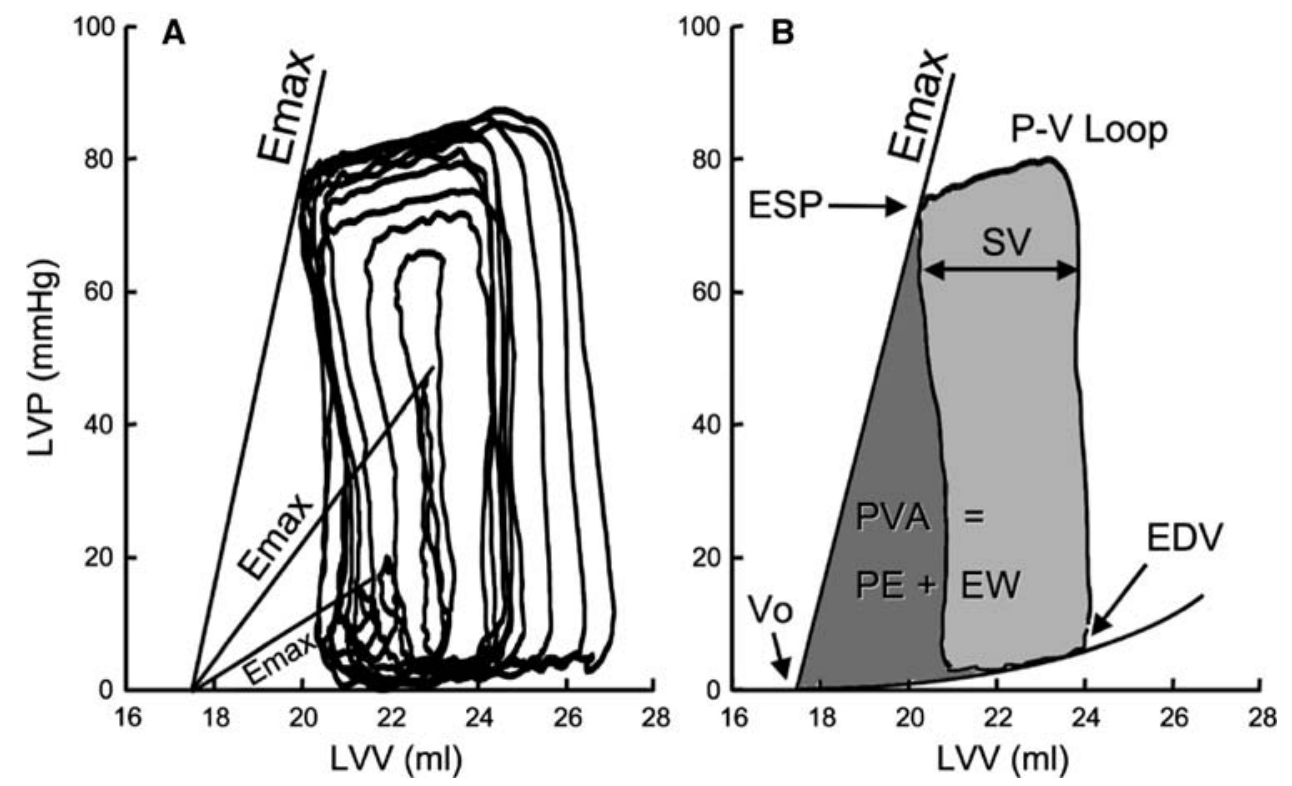

Fig. 1 a shows the left ventricular $(L V)$ pressure $(P)$-volume $(V)$ loops of 14 arbitrarily chosen continuous arrhythmic contractions under electrically maintained atrial fibrillation in a canine heart. b shows one of the $P-V$ loops to explain abbreviations. In a, three $P-V$ loops show their $\mathrm{E}_{\max }$ values as the slopes of the respective endsystolic $P-V$ lines drawn from the common dead volume on the volume axis. In $\mathbf{b}$, the area within the quasi-rectangular $P-V$ loop, mostly shaded, is the external work $(E W)$, and the quasi-triangular

$V \mathrm{O}_{2}=a \mathrm{PVA}+b \mathrm{E}_{\max }+c$

where $a=\mathrm{O}_{2}$ cost of PVA, $b=\mathrm{O}_{2}$ cost of $\mathrm{E}_{\max }$, and $c=$ basal metabolic $V \mathrm{O}_{2}$ of each beat, all normalized for $100 \mathrm{~g} \mathrm{LV}$. We already established the reasonable $\mathrm{VO}_{2}$ predictability of this equation for a stable regular beat [1] and proposed the $V \mathrm{O}_{2}$ per beat prediction method for each individual arrhythmic beat [4]. Although the $\mathrm{VO}_{2}$ predictability for a stable regular beat is solid empirically [1], that of an arrhythmic beat is not yet so because no direct method is yet available to confirm it on a per-arrhythmicbeat basis.

As the representative $a$ and $b$ values of canine LVs, we used $a=1.8 \times 10^{-5} \mathrm{ml} \mathrm{O} /(\mathrm{mmHg} \mathrm{ml})$ and $b=1.0 \times$ $10^{-3} \mathrm{ml} \mathrm{O}_{2} /(\mathrm{mmHg} / \mathrm{ml})[1,9]$. We could reasonably assume these $a$ and $b$ values to be independent of the LV loading and contractile conditions (1). As for the $c$ value, we used $c=1.0 \times \mathrm{RR} / 60,000 \mathrm{ml} \mathrm{O} /$ beat, all per $100 \mathrm{~g}$ LV. Here, 1.0 is in $\mathrm{ml} \mathrm{O}_{2} / \mathrm{min}$, and both RR (ECG R-R interval) and 60,000 are in ms. Since these representative values have some variations even among normal canine hearts (1), the above calculated $V \mathrm{O}_{2}$ values could serve as representatively estimated values in normal canine LVs. area, also mostly shaded, under the $\mathrm{E}_{\max }$ line on the origin side of the $\mathrm{EW}$ area is the mechanical potential energy $(P E)$. The systolic pressure-volume area $(P V A)$ is the sum of these two areas, namely, $\mathrm{PVA}=\mathrm{PE}+\mathrm{EW}$. The stroke volume $(S V)$ is the width of the $P-V$ loop; the end-systolic pressure $(E S P)$ is the pressure at the left upper corner of the $P-V$ loop; the end-diastolic volume $(E D V)$ is the volume at the right lower corner of the $P-V$ loop. $V_{0}$ is the dead volume at which end-systolic pressure is zero

The EW efficiency is defined as $\mathrm{EW} / V \mathrm{O}_{2}$. We obtained EW as the area within each arrhythmic $P-V$ loop (Fig. 1b). We divided it by the above-estimated $V \mathrm{O}_{2}$. Namely,

$\mathrm{EW} / V \mathrm{O}_{2}=\mathrm{EW} /\left(a \mathrm{PVA}+b \mathrm{E}_{\max }+c\right)$

EW in $\mathrm{mmHg} \mathrm{ml}$ was converted to EW in joule (J), using $1 \mathrm{mmHg} \mathrm{ml}=1.33 \times 10^{-4} \mathrm{~J} . V \mathrm{O}_{2}$ in $\mathrm{ml} \mathrm{O}_{2}$ was also converted to $V_{2}$ in $\mathrm{J}$ by $1 \mathrm{ml} \mathrm{O}_{2}=20 \mathrm{~J}$ (1). Therefore, $\mathrm{EW} / V \mathrm{O}_{2}$ became dimensionless between 0 and 1 or percent between 0 and $100 \%$.

Statistical analyses

We analyzed the basic statistics $[10,11]$ of the obtained mechanoenergetic variables of individual arrhythmic beats sampled for $1 \mathrm{~min}$ in each of the six hearts. We had shown that $1 \mathrm{~min}$ was long enough to obtain a large enough number of arrhythmic beats for statistical analyses since we had found both $\mathrm{E}_{\max }$ and PVA to distribute normally even within 1 min [8]. We used Microsoft Office 2004 Excel and SAS StatView 5.0 for the statistical analyses. 


\section{Results}

Figure 1 shows a representative set of continuous LV $P-V$ loops of arrhythmic beats under AF in one canine heart (Panel a) and a representative one of these loops (Panel b). The loops clearly show that their $\mathrm{E}_{\max }$ fluctuated among the arrhythmic beats, and their SV, EW, PE, PVA $(=\mathrm{PE}+\mathrm{EW}), \mathrm{EDV}$, and ESP also fluctuated simultaneously. As for these fluctuations under $\mathrm{AF}$, we have already analyzed them in detail [4, 5, 8, 12-15].

Figure 2 plots LV EW (open circles) and its efficiency (solid circles) against the predicted $\mathrm{LV} \mathrm{VO}_{2}$ of arrhythmic beats for $30 \mathrm{~s}$ in one canine heart. The $\mathrm{EW}-V \mathrm{O}_{2}$ data fell fairly linearly with an extrapolated $V \mathrm{O}_{2}$ intercept of $\sim 0.3 \mathrm{~J} / 100 \mathrm{~g}$ per beat. Since the mean arrhythmic heart rate was $\sim 200$ beats/min, this extrapolated $V \mathrm{O}_{2}$ intercept per beat corresponds to $\sim 60 \mathrm{~J} / 100 \mathrm{~g}$ per min. Although this $V \mathrm{O}_{2}$ intercept was about three times a representative basal metabolic rate of $1 \mathrm{ml} \mathrm{O} 2 / \mathrm{min}$ or $20 \mathrm{~J} / 100 \mathrm{~g} / \mathrm{min}$ under $\mathrm{KCl}$ arrest, we consider it physiologically reasonable because it was comparable to a representative PVA-independent $\mathrm{VO}_{2}$ of $0.3 \mathrm{~J} / 100 \mathrm{~g}$ per regular beat in a relatively low $\mathrm{E}_{\max }(1)$, consisting of both basal metabolic $V \mathrm{O}_{2}$ and excitation-contraction coupling $V \mathrm{O}_{2}$. Similar $\mathrm{EW}-\mathrm{VO}_{2}$ relations were observed in all the other hearts.

The EW efficiency corresponds to the slope of the line connecting the respective $\mathrm{EW}-\mathrm{VO}_{2}$ data (open circle) to the origin, but not to the positive $V \mathrm{O}_{2}$ intercept, indicating the PVA-independent $V \mathrm{O}_{2}$. As the result, the EW efficiency tended to saturate as $\mathrm{VO}_{2}$ increased. Similar EW efficiency$V \mathrm{O}_{2}$ relations were observed in all the other hearts.

Figure 3 shows the frequency distribution histogram of the EW efficiency of the same data used in Fig. 2. The histogram obviously appeared to skew rightward as

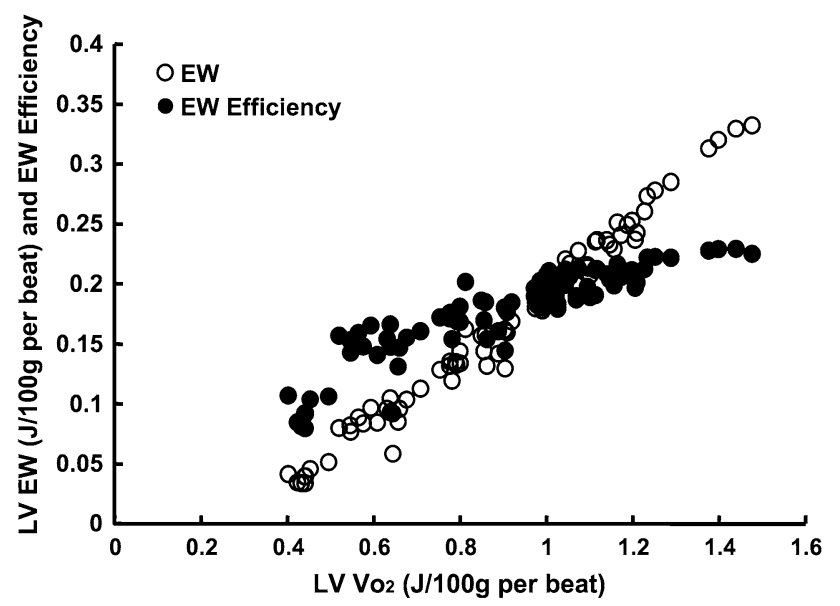

Fig. 2 Scattergram of left ventricular $(L V)$ external work (EW) (open circles) and work efficiency (solid squares, dimensionless) on the ordinate against $\mathrm{LV} \mathrm{O}_{2}$ consumption $\left(V \mathrm{O}_{2}\right)$ on the abscissa in one canine heart under electrically induced atrial fibrillation compared to the normal distribution curve, although $\chi^{2}$ was not statistically significant $(P>0.05)$. Similar distributions of the EW efficiency were observed in all the other hearts.

Figure 3 evidently shows that the mode EW efficiency of the arrhythmic beats was comparable to the constant EW efficiency of the regular beats and the maximum EW efficiency was variably greater than the stable EW efficiency of the regular beats $(P<0.01$, paired $t$ test).

Table 1 compares the EW efficiency data of the arrhythmic beats under AF and regular beats in each of the six hearts. Paired comparisons between them also showed that the maximum efficiency was significantly greater than the efficiency of regular beats in every heart as well as on average and the mode efficiency was comparable to the regular beat efficiency.

However, the mean efficiency was significantly smaller than the regular beat efficiency in every heart as well as on average (Fig. 3; Table 1).

\section{Discussion}

This is the first study to have revealed the statistical characteristics of the distribution of EW efficiency of individual arrhythmic beats under electrically induced AF in non-failing hearts, though canine hearts. The mode EW efficiency (15\% on average) of the LV arrhythmic beats under electrically induced AF was comparable to the mean EW efficiency $(15 \%)$ of the regular beats in the six normal canine in situ hearts (Table 1). Moreover, the maximum EW efficiency (18\% on average) under AF was greater than the mean EW efficiency of the regular beats in these hearts (Table 1).

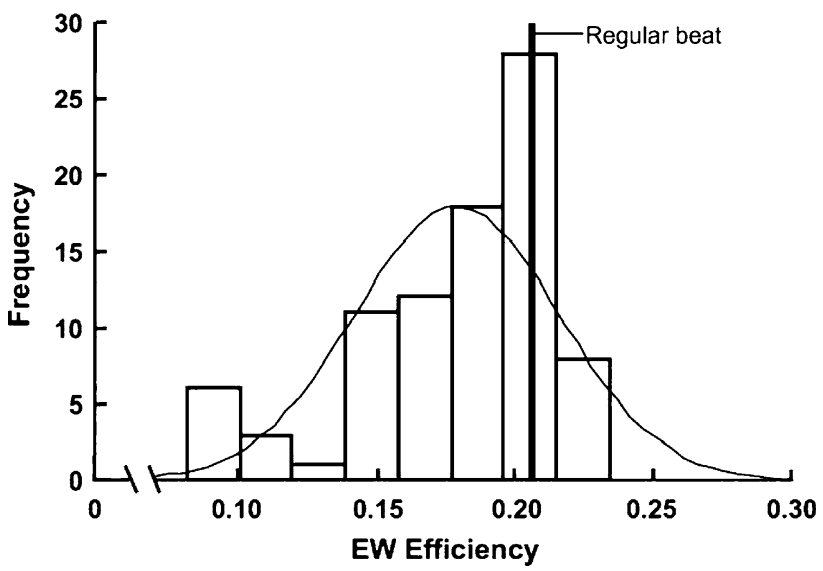

Fig. 3 Frequency distribution histogram of external work $(E W)$ efficiency of arrhythmic beats under atrial fibrillation in a canine heart skewed to the higher efficiency and exceeded the mean EW efficiency of regular beats 
Table 1 Comparison of external work efficiency between arrhythmic and regular beats under atrial fibrillation in six hearts

\begin{tabular}{|c|c|c|c|c|c|}
\hline \multirow[t]{2}{*}{ Heart } & \multicolumn{4}{|l|}{ Arrhythmic } & \multirow[t]{2}{*}{ Regular } \\
\hline & Minimum & Mean & Mode & Maximum & \\
\hline 1 (relative) & $0.045(35 \%)$ & $0.107(83 \%)$ & $0.120(93 \%)$ & $0.138(107 \%)$ & $0.129(100 \%)$ \\
\hline 2 (relative) & $0.068(50 \%)$ & $0.132(98 \%)$ & $0.145(107 \%)$ & $0.161(119 \%)$ & $0.135(100 \%)$ \\
\hline 3 (relative) & $0.065(41 \%)$ & $0.116(73 \%)$ & $0.125(78 \%)$ & $0.178(111 \%)$ & $0.160(100 \%)$ \\
\hline 4 (relative) & $0.037(27 \%)$ & $0.119(87 \%)$ & $0.145(106 \%)$ & $0.190(139 \%)$ & $0.137(100 \%)$ \\
\hline 5 (relative) & $0.036(24 \%)$ & $0.145(96 \%)$ & $0.155(106 \%)$ & $0.191(127 \%)$ & $0.151(100 \%)$ \\
\hline 6 (relative) & $0.081(39 \%)$ & $0.179(87 \%)$ & $0.205(100 \%)$ & $0.232(113 \%)$ & $0.206(100 \%)$ \\
\hline Efficiency mean & 0.055 & 0.133 & 0.149 & 0.182 & 0.153 \\
\hline SD & 0.019 & 0.026 & 0.030 & 0.032 & 0.028 \\
\hline Relative mean & $36 \%$ & $88 \%$ & $99 \%$ & $122 \%$ & $100 \%$ \\
\hline SD & $11 \%$ & $10 \%$ & $12 \%$ & $11 \%$ & $0 \%$ \\
\hline$P$ for paired $t$ & $<0.0001$ & $<0.05$ & $>0.5(\mathrm{~ns})$ & $<0.01$ & Control \\
\hline
\end{tabular}

Arrhythmic columns list minimum, mean, mode (i.e., most frequent), and maximum efficiencies of arrhythmic beats under atrial fibrillation in comparison with constant efficiency of regular beats in each of six left ventricles. Paired t tests were applied between each of minimum, mean, mode, and maximum efficiencies of arrhythmic beats and efficiency of regular beats in the respective hearts. $n s: P>0.05$

As Fig. 2 indicates, EW can be expressed as a linear function as

$\mathrm{EW}=p V \mathrm{O}_{2}+q$.

Here, $p$ is the slope of the $V \mathrm{O}_{2}$-EW relation $(p>0)$ and $q$ is the extrapolated EW axis intercept of the relation $(q<0)$. So, the EW efficiency can be expressed as

$\mathrm{EW} / V \mathrm{O}_{2}=p+q / V \mathrm{O}_{2}$.

As seen in Fig. 2, the EW efficiency showed the hyperbolic relation. This hyperbolic relation means the higher EW efficiency at the beat consuming the more oxygen. The short RRs in both regular tachycardia and AF, both decreasing the contractile force and shortening the filling time, decrease PVA, EW, and $V \mathrm{O}_{2}$ per beat. Thus, this hyperbolic relation between $V \mathrm{O}_{2}$ and $\mathrm{EW}$ efficiency indicates that the shorter RR causes the lower EW efficiency and may account for the reason of the beneficial effect of the rate control therapy for patients suffering from AF $[16$, 17].

The mean EW efficiency (13\%) of the arrhythmic beats was, however, significantly smaller than the stable EW efficiency $(15 \%)$ of the regular beats (Table 1). This confirms the already known experimental and clinical findings [18-21].

These mean, mode, and maximum efficiencies of the arrhythmic beats were also within the normal working ranges found not only in our previous studies on normal canine hearts [1, 22, 23], but also in other studies [24].

There are some limitations in this study. We were unable to compare $\mathrm{EW} / \mathrm{VO}_{2}$ at the same heart rate between the irregular beats under $\mathrm{AF}$ and the regular beats under the regular atrial pacing. The latter was $22 \%$ lower than the former. This was simply because the LV started to have pulsus alternans already at a pacing rate before reaching the mean arrhythmic heart rate under AF. Therefore, we cannot yet state that the present results would hold if the pacing rate is matched with the mean arrhythmic heart rate under AF.

An obvious limitation of the present study existed in the LV volumetry with the conductance catheter. The LV conductance changed even in the isovolumic contraction and relaxation phases due to the deformation of the LV cavity, as seen in Fig. 1. Therefore, even when no SV and hence no EW is produced in a relatively weak contraction, the $P-V$ loop is a slightly open loop, but not a vertical line with zero width. For this reason, the minimum EWs even of weak arrhythmic beats presumably without SV were not zero as shown in Figs. 1 and 2. Therefore, the minimum EW efficiency was not zero as shown by the leftmost slightly high histogram bar in Fig. 3 .

To evaluate the effect of this volume error, we zeroed the efficiency values of the leftmost histogram bar and reanalyzed the frequency distribution. As the result, the minimum EW efficiency could be zero, and the mean value would decrease in Table 1. Although this correction could increase the absolute value of the negative skewness and the positive kurtosis and augment the non-normality, the mode and maximum values in Table 1 remained unchanged.

Another limitation is that the present study was performed in normal, but not failing hearts. Therefore, the present finding cannot simply be extrapolated to variably failing hearts.

Taken together, only the mode, but not the mean, minimum, and maximum of the left ventricular EW efficiency 
of arrhythmic beats under AF remained comparable to the stable efficiency at the regular tachycardia, which was, however, slightly lower than the mean arrhythmic tachycardia in the normal canine hearts. We reasonably accounted for these statistical findings mentioned above by the ratio of the normally distributing $\mathrm{EW}$ of each beat under $\mathrm{AF}$ to the respective $\mathrm{VO}_{2}$ linearly correlating with $\mathrm{EW}$, but always containing a considerable PVA-independent $V \mathrm{O}_{2}$ consisting of both basal metabolic $V \mathrm{O}_{2}$ and excitation-contraction coupling $V \mathrm{O}_{2}$. These cardiac energetic properties should be recognized to elucidate the mechanisms of the decreased cardiac performance under AF.

Acknowledgments We appreciated Hiroki Yamaguchi, $\mathrm{MD}, \mathrm{PhD}$, for his surgical contribution to this series of experiments in Okayama University. This work was partly supported by Scientific Research Grants nos. 13558113, 13770350, 13878185, 13878192, 14380405, 15650095, 15659186, 16659057, 17390230, 17390231, 18650125, and 20300162 from the Ministry of Education, Culture, Sports, Science and Technology, and Cardiovascular Diseases Research Grants (11C-1, 14A-1) from the Ministry of Health, Labor, and Welfare, both of Japan.

\section{References}

1. Suga H (1990) Ventricular energetics. Physiol Rev 70:247-277

2. Sagawa K, Maughan L, Suga H, Sunagawa K (1988) Cardiac contraction and the pressure-volume relationship. Oxford Univ Press, New York, pp 232-298

3. Suga H, Sagawa K, Shoukas AA (1973) Load independence of the instantaneous pressure-volume ratio of the canine left ventricle and effects of epinephrine and heart rate on the ratio. Circ Res 32:314-322

4. Shimizu J, Mohri S, Iribe G, Ito H, Morita T, Yamaguchi H, Sano $\mathrm{S}$, Suga $\mathrm{H}$ (2005) Predictability of $\mathrm{O}_{2}$ consumption from contractility and mechanical energy of absolute arrhythmic beats in canine heart. Jpn J Physiol 55:135-142. doi:10.2170/jjphysiol. R2099

5. Yamaguchi H, Takaki M, Ito H, Tachibana H, Lee S, Suga H (1997) Pressure-interval relationship characterizes left ventricular irregular beat contractilities and their mean level during atrial fibrillation. Jpn J Physiol 47:101-110. doi:10.2170/jjphysiol.47. 101

6. Baan J, van der Velde ET, de Bruin HG, Smeenk GJ, Koops J, van Dijk AD, Temmerman D, Senden J, Buis B (1984) Continuous measurement of left ventricular volume in animals and humans by conductance catheter. Circulation 70:812-823

7. Suga H, Hisano R, Ninomiya I (1982) Digital on-line computation of a predictor of cardiac oxygen consumption. Left ventricular systolic pressure volume area. Jpn Heart J 23:749-758

8. Morita T, Araki J, Oshima Y, Mitani H, Iribe G, Mohri S, Shimizu J, Sano S, Kajiya F, Suga H (2002) Frequency distribution, variance, and moving average of left ventricular rhythm and contractility during atrial fibrillation in dog. Jpn J Physiol 52:4149. doi:10.2170/jjphysiol.52.41
9. Ohgoshi Y, Goto Y, Futaki S, Yaku H, Kawaguchi O, Suga H (1990) New method to determine oxygen cost for contractility. Jpn J Physiol 40:127-138. doi:10.2170/jjphysiol.40.127

10. Snedecor G, Cochran W (1971) Statistical methods, 6th edn. Ames, Iowa, Iowa State Univ Press

11. Glantz S. (1981) Primer of biostatics. McGraw-Hill Book Co. New York, pp 217-221, 284-292

12. Mohri S, Shimizu J, Iribe G, Ito H, Morita T, Yamaguchi H, Sano S, Suga H (2005) Normal distribution of ventricular pressurevolume area (PVA) of arrhythmic beats under atrial fibrillation in canine heart. Am J Physiol Heart Circ Physiol 288:H1740 H1746. doi:10.1152/ajpheart.00584.2004

13. Mitani H, Yamaguchi H, Morita T, Oshima Y, Shimizu J, Ito H, Araki J, Takaki M, Sano S, Suga H (2000) Effective arterial elastance of irregular beats during atrial fibrillation in canine left ventricle. Jpn J Physiol 50:77-89. doi:10.2170/jjphysiol.50.77

14. Shimizu J, Mohri S, Ito H, Yamaguchi H, Sano S, Takaki M, Suga H (2006) High mechanical efficiency of left ventricular arrhythmic contractions during atrial fibrillation. J Physiol Sci 56:269-274. doi:10.2170/physiolsci.RP004206

15. Mohri S, Shimizu J, Ito H, Yamaguchi H, Sano S, Takaki M, Suga H (2005) Variable unstressed volume keeps normal distributions of canine left ventricular contractility and total mechanical energy under atrial fibrillation. Jpn J Physiol 55:255264. doi:10.2170/jjphysiol.RP000405

16. Savelieva I, Camm AJ (2004) Atrial fibrillation and heart failure: natural history and pharmacological treatment. Europace 5:S5S19. doi:10.1016/j.eupc.2004.07.003

17. The Atrial fibrillation follow-up investigation of rhythm management (AFFIRM) investigators (2002) A comparison of rate control and rhythm control in patients with atrial fibrillation. $\mathrm{N}$ Engl J Med 347:1825-1833

18. Brookes CI, White PA, Staples M, Oldershaw PJ, Redington AN, Collins PD, Noble MI (1998) Myocardial contractility is not constant during spontaneous atrial fibrillation in patients. Circulation 98:1762-1768

19. Kerr AJ, Simmonds MB, Stewart RA (1998) Influence of heart rate on stroke volume variability in atrial fibrillation in patients with normal and impaired left ventricular function. Am J Cardiol 82:1496-1500. doi:10.1016/S0002-9149(98)00693-6

20. Muntinga HJ, Gosselink AT, Blanksma PK, De Kam PJ, Van Der Wall EE, Crijns HJ (1999) Left ventricular beat to beat performance in atrial fibrillation: dependence on contractility, preload, and afterload. Heart 82:575-580

21. Yamada H, Mowrey KA, Popovic ZB, Kowalewski WJ, Martin DO, Thomas JD, Wallick DW (2004) Coupled pacing improves cardiac efficiency during acute atrial fibrillation with or without cardiac dysfunction. Am J Physiol Heart Circ Physiol 287:H2016-H2022. doi:10.1152/ajpheart.00347.2004

22. Suga H, Igarashi Y, Yamada O, Goto Y (1985) Mechanical efficiency of the left ventricle as a function of preload, afterload, and contractility. Heart Vessels 1:3-8. doi:10.1007/BF02066480

23. Nozawa T, Yasumura Y, Futaki S, Tanaka N, Uenishi M, Suga H (1988) Efficiencies from consumed $\mathrm{O}_{2}$ and pressure-volume area to work of in situ dog heart. Jpn J Physiol 38:713-728. doi: 10.2170/jjphysiol.38.713

24. Gibbs CL, Barclay CJ (1995) Cardiac efficiency. Cardiovasc Res 30:627-634

25. Sunagawa K, Maughan WL, Burkhoff D, Sagawa K (1983) Left ventricular interaction with arterial load studied in isolated canine heart. Am J Physiol 245:H773-H780 